\title{
Preparation and Identification of Per a 5 as a Novel American Cockroach Allergen
}

\author{
Ji-Fu Wei, ${ }^{1,2}$ Haiwei Yang, ${ }^{3}$ Dongning Li, ${ }^{1}$ Peisong Gao, ${ }^{4}$ and Shaoheng He ${ }^{1,2}$ \\ ${ }^{1}$ Allergy and Clinical Immunology Research Centre, The First Affiliated Hospital of Liaoning Medical University, \\ Jinzhou, Liaoning 121001, China \\ ${ }^{2}$ Research Division of Clinical Pharmacology, The First Affiliated Hospital of Nanjing Medical University, \\ Nanjing, Jiangsu 210029, China \\ ${ }^{3}$ Department of Urology, The First Affiliated Hospital of Nanjing Medical University, Nanjing, Jiangsu 210029, China \\ ${ }^{4}$ Johns Hopkins Asthma \& Allergy Center, Baltimore, MD 21224, USA \\ Correspondence should be addressed to Shaoheng He; shoahenghe@hotmail.com
}

Received 24 November 2013; Revised 15 January 2014; Accepted 15 January 2014; Published 23 February 2014

Academic Editor: Huiyun Zhang

Copyright @ 2014 Ji-Fu Wei et al. This is an open access article distributed under the Creative Commons Attribution License, which permits unrestricted use, distribution, and reproduction in any medium, provided the original work is properly cited.

\begin{abstract}
Glutathione S-transferase (GST) from various arthropods can elicit allergic reactions. In the present study, Per a 5, a GST, was cloned from American cockroach (CR) and expressed in both baculovirus-infected insect cell (iPer a 5) and E. coli expression (bPer a 5) systems. The secondary structures were predicted to be 45.93 and $8.69 \%$ of $\alpha$-helix $\beta$-sheets in iPer a 5 and 42.54 and $8.49 \%$ of $\alpha$-helix and $\beta$-sheets in bPer a 5, respectively. It is found that 4 out of 16 (25\%) sera from American CR allergy patients reacted to both bPer a 9 and iPer a 9 as assessed by ELISA and Western blotting analysis, confirming that Per a 5 is not a major allergen of American CR. Induction of upregulated expression of CD63 and CCR3 on passively sensitized human basophils (sera from American CR allergy patients) by approximately up to 4.5- and 3.2-fold indicates that iPer a 5 and bPer a 5 are functionally active. Recombinant Per a 5 (rPer a 5) should be a useful tool for studying and understanding the role of Per a 5 in CR allergy.
\end{abstract}

\section{Introduction}

CR allergy has been recognized as important IgE-mediated type I hypersensitivity since 1964 [1]. It is associated with the development of asthma and recognized as a risk factor for emergency room admission of asthmatic patients, especially among inner city children living in low-income houses infested with cockroaches [2]. In China, totally $25.7 \%$ of allergy patients are skin prick test (SPT) positive to the American CR (Periplaneta americana, Per a) and 18.7\% SPT positive to the German CR (Blattella germanica, Bla g) [3].

American CR, German CR, and smoky brown CR (Periplaneta fuliginosa) are the dominant indoor CR species which cause allergy among human population worldwide [2] . Twenty-two immunoglobulin E (IgE) binding components including the proteins of $23,28,35,38,40,49,72,78$, and $97 \mathrm{kDa}$ were identified as major allergens in American CR [4], but only Per a 1 [5], Per a 2 [6], Per a 3 [7], Per a 4 [8], Per a 7 [9], Per a 9 [10], and Per a 10 [11] are characterized.
For example, Per a 1 is an isoallergen with 5 isoforms reported so far, that is, Per a 1.0101-Per a 1.0105. Per a 1 is a major American CR allergen as it binds to IgE in the sera of 90$100 \%$ of CR allergic subjects [4]. Per a 2 is an inactive aspartic protease found in the American CR digestive tract and feces [5]. Per a 5 belongs to GSTs, but the allergenicity of the GST homologues in American CR has not been reported. Since the GST of German CR (Bla g 5) is a major cockroach allergen, which induces specific IgE expression in 30 to $71 \%$ of CR allergy [12-14], we anticipate that Per a 5 is likely a major allergen of American CR and investigate the potential allergenicity of it in the present study.

Two GST homologue (Per a 5) genes are available in the Genbank (Accession: AY792949 and AEV23867), which provides the possibility of producing recombinant Per a 5 (rPer a 5) in large amounts to study its role in allergic reactions. Many, but not all allergens expressed from cDNA have shown a considerable IgE binding reactivity that seems to be comparable to their natural counterparts. The majority 
of these recombinant allergens are produced in E. coli, but, unfortunately, the amount and/or reactivity is sometimes reduced when the allergen is purified and subjected to immunological and biochemical assays [15]. To overcome some of these problems, eukaryotic expression systems such as yeast and baculovirus in insect cells have been used [16]. The aim of the present study is to generate rPer a 5 by using eukaryotic (baculovirus-infected insect cells) and prokaryotic (E. coli) expression systems and characterize its biochemical and immunologic properties.

\section{Materials and Methods}

2.1. Ethics Statement. The study protocol was approved by the Ethical Committee of the First Affiliated Hospital of Nanjing Medical University. Written informed consent for the use of blood samples was obtained from all participants before study entry according to the declaration of Helsinki.

2.2. Patients and Samples. A total of 16 allergic rhinitis patients with positive SPT (allergens were supplied by ALKAbelló, Inc., Denmark) and positive serum IgE test to American CR extract (by using Immuno-CAP assay (Pharmacia Diagnostics AB, Uppsala, Sweden)) and 6 healthy controls (HC) were recruited in the study. Serum $(4 \mathrm{~mL})$ from peripheral venous blood was collected from each patient and $\mathrm{HC}$ for Western blot analysis.

2.3. Cloning of cDNAs Encoding Full Length of Per a 5 Gene. Total RNA was isolated from adult female CR reared at our institute by using TRIzol reagent (Invitrogen, Carlsbad, CA, USA). Total RNA was quantified by measuring absorbance ratios at $260 / 280 \mathrm{~nm}$. The cDNA was prepared by reverse transcription using a commercial RNA-PCR kit according to the manufacturer's instruction (TaKaRa Biotech Co. Ltd., Dalian, China). For each reaction, $1 \mu \mathrm{g}$ of total RNA was reversely transcribed using oligo-d (T). The cDNAs encoding Per a 5 were amplified by PCR using primers based nucleotide sequence of Per a 5 gene (AY792949 and AEV23867; forward: 5'-ATGACCATCGACTTCTACTA$3^{\prime}$; reverse: $5^{\prime}$-TCACTTCTTGGCGAGGTTAT-3 ${ }^{\prime}$ ). PCR condition was $95^{\circ} \mathrm{C} / 5 \mathrm{~min}$ (one cycle), $95^{\circ} \mathrm{C} / 1 \mathrm{~min}$, $52^{\circ} \mathrm{C} / 1 \mathrm{~min}$ and $72^{\circ} \mathrm{C} / 1 \mathrm{~min}$ (30 cycles), and $72^{\circ} \mathrm{C} / 5 \mathrm{~min}$ (one cycle). The purified PCR product was cloned into apMD18-T vector (TaKaRa Biotech Co. Ltd., Dalian, China) before being transformed into Escherichia coli strain DH5 $\alpha$. The inserts were sequenced on an ABIprism 377 DNA sequencer (Applied Biosystems, Foster, CA, USA). DNA sequence data were translated to amino acid sequence by Show Translation tool in SMS software package (http://www.bioinformatics.org/SMS/). The glycosylation motifs of Per a 5 were predicted by using NetNGlyc 1.0 Server (http://www.cbs.dtu.dk/services/NetNGlyc).

2.4. Expression and Purification of Per a 5 in BaculovirusInfected Insect Cells. The Per a 5 gene was subcloned into pFastBacl vector (Novagen, Madison, WI, USA) using EcoR I and Sal I sites and the resulted construct was transformed into
E. coli strain DH10Bac to generate recombinant bacmid. The positive colonies were selected and followed by PCR identification. The recombinant bacmid was transfected into Sf-9 cells by using Cellfectin (Invitrogen Corporation, Carlsbad, USA) and incubated in SF-900II liquid medium (Invitrogen Corporation, Carlsbad, USA) for 5 days at $27^{\circ} \mathrm{C}$ until the cells got swollen. The supernatant was collected as $\mathrm{P} 1$ viral stock. P2 viruses were amplified for later infection. A total of $500 \mathrm{~mL}$ of Sf- 9 cells were infected by P2 viruses and harvested at $72 \mathrm{~h}$. The cells were lysed against $50 \mathrm{mM}$ Tris- $\mathrm{HCl}$ with $300 \mathrm{mM} \mathrm{NaCl}$ and 5\% glycerol. The supernatant was loaded on Ni-NTA column (Genscript, Nanjing, China), washed with running buffer containing $50 \mathrm{mM}$ Tris- $\mathrm{HCl}, 300 \mathrm{mM}$ $\mathrm{NaCl}$, and $5 \%$ glycerol $(\mathrm{pH} 8.0)$ and eluted with elution buffer containing $50 \mathrm{mM}$ Tris- $\mathrm{HCl}, 300 \mathrm{mM} \mathrm{NaCl}, 250 \mathrm{mM}$ imidazole, and 5\% glycerol $(\mathrm{pH} 8.0)$. The eluted fractions were obtained and identified as Per a 5 (iPer a 5). The purified iPer a 5 was dialyzed in carbonate-bicarbonate buffer $(0.05 \mathrm{M}$, $\mathrm{pH}$ 9.6) for further investigation. The concentration of iPer a 5 was determined by using a Coomassie Plus assay kit with BSA as standard (Thermo Scientific Pierce, Rockford, IL, USA).

2.5. Expression and Purification of Per a 5 in E. Coli. The Per a 5 gene was subcloned into pCold II vector (TaKaRa Biotech Co. Ltd., Dalian, China) using Nde I and Xba I sites and verified by DNA sequencing. The recombinant pCold IIPer a 5 plasmid was transformed into E. coli Origami host strain. A colony of the selected transformed E. coli Origami on an overnight LB-ampicillin agar plate was inoculated into $5 \mathrm{~mL}$ of LB-ampicillin broth and incubated at $37^{\circ} \mathrm{C}$ overnight. For IPTG optimization, the overnight culture was added into fresh LB media in a ratio of $1: 100$. Once cell density reached the optical density at $A_{600 \mathrm{~nm}}\left(\mathrm{OD}_{600}\right)$, the cells were incubated with $0.1,0.5$, and $1 \mathrm{mM}$ IPTG, respectively, at $15^{\circ} \mathrm{C}$ overnight. Expression of the target protein was analyzed by SDS-PAGE. For scale-up expression, $40 \mathrm{~mL}$ of the culture was inoculated into $2 \mathrm{~L}$ of fresh LB-ampicillin broth and incubated at $37^{\circ} \mathrm{C}$ until $\mathrm{OD}_{600}$ reached 0.6. IPTG was added to the final concentration of $0.5 \mathrm{mM}$ and the culture was incubated overnight. The bacterial cells were harvested by centrifugation at $4,000 \times \mathrm{g}$ at $4^{\circ} \mathrm{C}$ for $20 \mathrm{~min}$ and were lysed in a lysis buffer by sonication at $20 \mathrm{kHz}, 2 \mathrm{~min}$ pulse-on, 3 min pulse-off. Cell debris was removed by centrifugation at $12,000 \times \mathrm{g}$ at $4^{\circ} \mathrm{C}$ for $20 \mathrm{~min}$. The supernatant was loaded on the Nickel column (Genscript, Nanjing, China) as described above, and the eluted fractions were obtained and identified as Per a 5 (bPer a 5). The purified bPer a 5 was dialyzed in carbonate-bicarbonate buffer $(0.05 \mathrm{M}, \mathrm{pH} 9.6)$ for further investigation. The concentration of bPer a 5 was determined by using a Coomassie Plus assay kit with BSA as standard.

2.6. CD Analysis of rPer a 5 Expressed in E. Coli and Insect Cells. Far UV CD spectra of bPer a 5 and iPer a 5 were collected on a Jasco J-810 spectropolarimeter (Japan Spectroscopic Co., Tokyo, Japan) using a $1 \mathrm{~mm}$ path length quartz cuvette at protein concentrations of $0.1 \mathrm{mg} / \mathrm{mL}$. Spectra were measured from 240 to $190 \mathrm{~nm}$, with a $0.5 \mathrm{~nm}$ resolution at a scanning speed of $50 \mathrm{~nm} / \mathrm{min}$, and resulted from averaging 
of three scans. All measurements were performed in $10 \mathrm{mM}$ $\mathrm{Na}_{2} \mathrm{HPO}_{4}, \mathrm{pH}$ 7.0. The final spectra were baseline corrected by subtracting the corresponding buffer spectrum. Results were expressed as the mean residue ellipticity $(y)$ at a given wavelength. The secondary structure content of bPer a 5 and iPer a 5 was calculated by using the secondary structure estimation program K2D2 [17].

2.7. Immunoreactivity of Human Sera with $r$ Per a 5. A 96well plate was coated with purified bPer a 5 and iPer a 5 at $10 \mu \mathrm{g} / \mathrm{mL}$ in carbonate-bicarbonate buffer $(0.05 \mathrm{M}, \mathrm{pH} 9.6)$ overnight at $4^{\circ} \mathrm{C}, 100 \mu \mathrm{L}$ per well. Human serum samples ( $1: 20$ dilution in PBS-Tween with 2\% BSA) were then added to the plates for $2 \mathrm{~h}$ at room temperature. After IgE binding, plates were incubated with horseradish peroxidase-labeled goat anti-human IgE (1:2500 dilution) (KPL, Inc., MD, USA), and the color was developed with tetramethylbenzidine peroxidase substrate (Thermo Scientific Pierce, Rockford, IL, USA). The plates were read on a microplate reader at absorbance of $405 \mathrm{~nm}$. The cutoff of the ELISA was calculated as the mean of the negative controls plus 2 SDs.

For competitive ELISA test, a 96-well plate was coated with American cockroach extract $(10 \mu \mathrm{g} / \mathrm{mL})$ in carbonatebicarbonate buffer $(0.05 \mathrm{M}, \mathrm{pH} 9.6)$ overnight at $4^{\circ} \mathrm{C}$. The 1:20-diluted pooled serum alone or preincubated with various quantities of the crude extract, iPer a 5 and bPer a 5 , for $2 \mathrm{~h}$ was added to the plates. The color development and plate reading procedure was the same as the previous section. Crude extract of cockroaches were prepared according to the methods described previously [18] with few modifications. Briefly, $30 \mathrm{~g}$ of cockroaches was pulverized in liquid nitrogen. The sample was defatted in $200 \mathrm{~mL}$ of ethyl ether and ethyl acetate $(1: 1$, by volume) and extracted with slow overhead stirring in carbonate-bicarbonate buffer $(0.05 \mathrm{M}, \mathrm{pH} 9.6)$, containing $6 \mathrm{mM} 2$-mercaptoethanol and 1/1,000 volume of protease inhibitor cocktail (Shenggong, Shanghai, China) at $4^{\circ} \mathrm{C}$ overnight. The extract was then centrifuged at $10,000 \times \mathrm{g}$ for $30 \mathrm{~min}$ at $4^{\circ} \mathrm{C}$, and the supernatant was filtered through a $0.22 \mu \mathrm{m}$-pore-size filter (Millipore, Bedford, USA) before use.

2.8. Immunoblot Analysis of IgE Reactivity. Immunoblots for detection of serum specific IgE were performed with bPer a 5 and iPer a 5 as described previously $[19,20]$. bPer a 5 and iPer a $5(5 \mu \mathrm{g})$ were added to a SDS-PAGE (gel concentration of $15 \%$ ) under reducing conditions and then transferred to nitrocellulose membranes. The nitrocellulose membranes were incubated with the sera from the patients with American CR allergy (1:5 in PBS-Tween with $1 \%$ BSA, $10 \%$ normal goat serum) for $90 \mathrm{~min}$. Following rinsing with PBS, the membranes were incubated with peroxidaselabeled anti-human IgE monoclonal antibody. The positive protein bands were visualized by incubating the membranes with tetramethylbenzidine peroxidase substrate. Sera from 2 nonatopic subjects were used as negative controls.

2.9. Basophil Activation Test. Expression of CD63 and CCR3 on basophil surface has been considered as the indicator of basophil activation [21, 22]. Briefly, peripheral blood mononucleated cells (PBMC) from $20 \mathrm{~mL}$ blood donated by 4 healthy volunteers were separated by Ficoll-Paque density gradient and treated with $10 \mathrm{~mL}$ LS (a solution containing $1.3 \mathrm{M} \mathrm{NaCl}, 0.005 \mathrm{M} \mathrm{KCl}$, and 0.01 lactic acid, at $\mathrm{pH} 3.9$ ) for $2 \mathrm{~min}$ at $8^{\circ} \mathrm{C}$. After neutralization with $12 \%$ Tris $(\mathrm{pH}$ 10.9), nonspecific IgE on basophils was stripped off and cells were passively sensitized with sera of the patients with American CR allergy or HC $(n=4,1$ in 10 dilution, $2 \mathrm{~h}$ at $37^{\circ} \mathrm{C}$ ) as described previously [23]. The cells were then challenged with various concentrations of bPer a 5 and iPer a 5 for $15 \mathrm{~min}$ at $37^{\circ} \mathrm{C}$. A goat anti-human $\operatorname{IgE}$ antibody (Serotec, Kidlington, UK) was used as a positive control. Anti-human CCR3-PE antibody (eBioscience Inc. San Diego, CA, USA) and anti-human CD63-FITC antibody (Invitrogen Corporation, Camarillo, CA, USA) were added to cells for $15 \mathrm{~min}$ at $37^{\circ} \mathrm{C}$ in the dark. Flow cytometry analysis of surface markers was performed at $488 \mathrm{~nm}$ on a FACSAria flow cytometer (Becton Dickinson, Franklin Lakes, NJ, USA) and analyzed by FACSDiva software. Basophils were gated in the lymphocyte region of the SSC or FSC pattern, and identified as a single population of cells that stained positively for CCR3-PE antibody. Upregulation of CD63 expression was determined by an increase in fluorescence in the FL-1 channel. Acquisition was terminated after 300 basophil target events. Responses were quantified as percentages of CD63 expressing basophils in a higher FL-1 region, which had been adjusted to contain $4 \%$ of basophils.

2.10. Statistics. Data are expressed as mean \pm SEM for the indicated number of independently performed duplicated experiments. Statistical significance between means was analyzed by one-way ANOVA or the Student's $t$-test utilizing the SPSS 13.0 version. $P<0.05$ was taken as statistically significant.

\section{Results}

3.1. Cloning of cDNAs Encoding Full Length of Per a 5 Sequence. The cDNAs encoding Per a 5 were amplified by PCR using primers based on the nucleotide sequence of Per a 5 gene. It is a 645 bp gene and encoded 215 amino acids protein (Figure 1). The sequence identity of Per a 5 to cDNAs deposited in Genbank (Accession no. AY563004) was 100\% (215/215) at protein level. Per a 5 shows 81,15 , and $13 \%$ sequence similarity to German CR allergen BGGSTD1, Der p 8, and Bla g 5 (Figure 2). One motif (198NHSG201) was predicted to be the glycosylation motif of Per a 5 .

3.2. Expression and Purification of Per a 5 in E. Coli. The Per a 5 gene was subcloned into pCold II vector and transformed into E. coli Origami host strain. The optimal induction condition for Per a 5 was 0.5 mM IPTG (Figure 3(a)), the concentration chosen as the final condition throughout the study. The Per a 5 protein was purified by Ni column. More than $6 \mathrm{mg}$ bPer a 5 was obtained from $2 \mathrm{~L}$ cell culture medium. The purity of the purified Per a 5 was identified by SDS-PAGE. It showed single band with an apparent molecular weight of $25 \mathrm{kDa}$ (Figure 3(b)). 


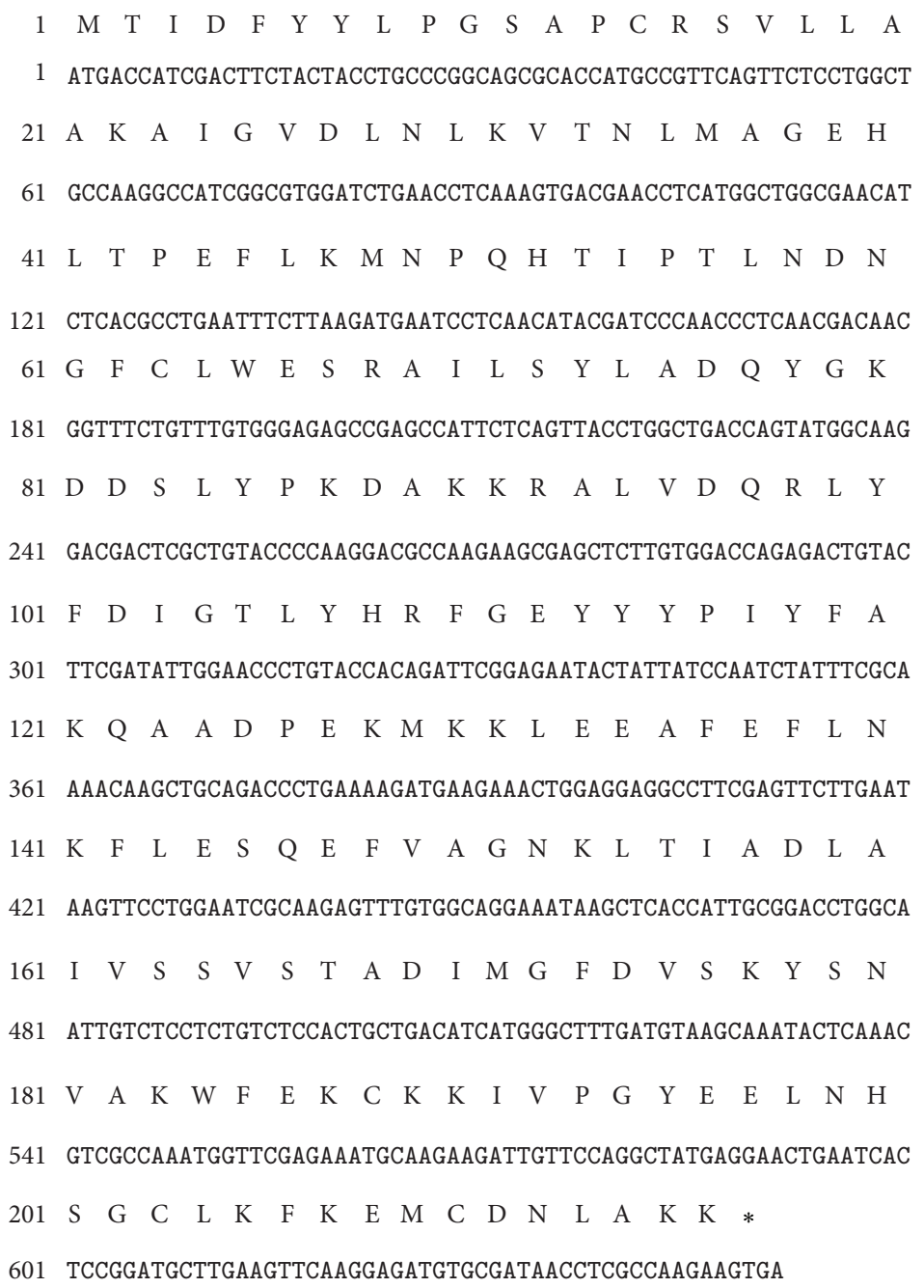

FIGURE 1: cDNA sequence encoding Per a 5 and deduced amino acid sequence. The first three bases ATG represent start codon. The last three bases indicate the stop codon.

3.3. Expression and Purification of Per a 5 in BaculovirusInfected Insect Cells. The Per a 5 encoding gene was subcloned into pFastBacl vector and transformed into $E$. coli strain $\mathrm{DH} 10 \mathrm{Bac}$ to generate recombinant bacmid. The recombinant bacmid was then transfected into Sf-9 cells to generate the baculovirus. The Per a 5 protein was expressed in Sf9 cells and purified by Ni column. About $16 \mathrm{mg}$ of iPer a 5 was obtained from $2 \mathrm{~L}$ cell culture medium. The purity of the purified Per a 5 was identified by SDS-PAGE, which showed major band with an apparent molecular weight of $25 \mathrm{kDa}$ (Figure 4).

3.4. CD Analysis of rPer a 5 Expressed in Insect Cells and E. Coli. The far UV CD spectra of iPer a 5 and bPer a 5 showed similar curves with two minima at 220 and $209.5 \mathrm{~nm}$ and a large maximum at $192 \mathrm{~nm}$, which represent characteristics of proteins with predominantly $\alpha$-helical structure (Figure 5). Calculation of the secondary structure using the program K2D2 resulted in predicted 45.93 and $8.69 \%$ of $\alpha$-helix and $\beta$-sheets in iPer a 5 and 42.54 and $8.49 \%$ of $\alpha$-helix and $\beta$ sheets in bPer a 5 , respectively.

3.5. Immunoreactivity to $I g E$. In order to determine the allergenicity of Per a 5, we examined the ability of Per a 5 to bind IgE in the sera of American CR allergy by a direct ELISA technique. Patients serum including patients 5, 9, 10, and 14 showed positive IgE reactivity to both bPer a 5 and iPer a 5 . The results showed that 4 out of 16 (25\%) sera from these patients reacted to both bPer a 5 and iPer a 5 (Figure 6(a)). The IgE reactivity of bPer a 5 and iPer a 5 in the sera from the Per a 5 positive patients was increased by 5.0 and 7.9-fold, respectively in comparison with the sera from HC. Moreover, competitive ELISA showed that bPer a 5 and iPer a 5 inhibited the IgE reactivity to the cockroach extract by approximately 25.4 and $35.5 \%$, respectively (Figure 6(b)). IgE binding activity of Per a 5 in a representative group of 3 patients and two $\mathrm{HC}$ was assessed by Western blot and was illustrated in Figure 6(c). IgE binding bands appeared clearer 


\begin{tabular}{|c|c|}
\hline er a 5 & --TIDFYYLPGSA-PCRSVLLAAKAIG-VDLNLKVT---NLMAGEHLTPEFLKMNPQHT \\
\hline BGGSTD1 & --TIDFYYLPGSA-PCRSVLLAAKAFG-VDLNLKVT---NLMAGEHLTPEFLKMNPQHT \\
\hline Der p 8 & SQPILGYWDIRGYAQPIR-LLLTYSGVDFVDKRYQIGPAPDFDRSEWLNEKFNLGLDFPN \\
\hline Blag a 5 & $\begin{array}{c}\text {--YKLTYCPVKALGEPIR-FLLSYGEKDFEDYRFQEG--------DWPN--LKPSMPFGK } \\
* * * * \quad *\end{array}$ \\
\hline Per a 5 & IPTLNDNGFCLWESRAILSYLADQYGKDDSLYPKDAKKRALVDQRLYFDIGTLYHRFGEY \\
\hline BGGSTD1 & IPTLNDNGFCLWESRAILSYLADQYGKDDSLYPKDPKKRALVDQRLYFDLGTLYQRFGDY \\
\hline Der $\mathrm{p} 8$ & LPYYIDGDMKMTQTFAILRYLGRKYKLNGSND-HEEIRISMAEQQTEDMMAAMI-R---V \\
\hline Blag a 5 & $\begin{array}{l}\text { TPVLEIDGKQTHQSVAISRYLGKQFGLSGKDD-WENLEIDMIVDTISDFRAAIAN--YHY } \\
* \quad * * * *\end{array}$ \\
\hline Per a 5 & YYPIYFAKQAADPEKMKKLEEAFEFLNKFLESQE-FVAGNKLTIADLAIVSSVSTADIMG \\
\hline BGGSTD1 & YYPIMFAKASPDAEKMKKLEEAYQFLDKFLEGQK-FVAGNSLTIADIATIASVSTAAILG \\
\hline Der p 8 & CYDANCDKLKPD--YLKSLPDCLKLMSKFVGEHA-FIAGANISYVDFNLYEYLCHVKVMV \\
\hline Blag a 5 & $\begin{array}{l}\text { DADENSKQKKWDPLKKETIPYYTKKFDEVVKANGGYLAAGKLTWADFYFVAILDYLNHMA } \\
* \quad 1 * *\end{array}$ \\
\hline Per a 5 & -FDVS-KYSNVAKWFEKCKKIVPGYEELNHSGCLKFKEMCDNLAKK-- $\quad 100 \%$ \\
\hline BGGSTD1 & -FDIT-RYPNVNKWFENAKKVIPGYDELNHSGCLEFKKMWDNLTQK-- $81 \%$ \\
\hline Der p 8 & -PEVFGQFENLKRYVERMESLPRVSDYIKKQQPKTFNAPTSKWNASYA $\quad 15 \%$ \\
\hline Blag a 5 & $\begin{array}{c}\text { KEDLVANQPNLKALREKVLGLPAIKAWVAKRPPTDL------------- } \\
\qquad * \quad * \quad 13 \%\end{array}$ \\
\hline
\end{tabular}

FIGURe 2: Alignment of Per a 5 with other GST allergen. Deduced amino acid sequence of Per a 5 is aligned with GST allergens. Per a 5 was cloned and sequenced in the present study. BGGSTD1 [29] and Bla g 5 [13] are two GST allergens identified in German CR, and Der p 8 is a GST allergen identified from Dermatophagoides pteronyssinus [30]. The identical residues in all four sequences are marked with asterisk $(*)$.

with iPer a 5 than with bPer a 5 . Both iPer a 5 and bPer a 5 did not react to the sera from the HC.

3.6. Induction of Basophil Activation by rPer a 5. iPer a 5 and bPer a 5 at $1.0 \mu \mathrm{g} / \mathrm{mL}$ induced approximately up to 4.5 and 3.2-fold increase in the expression of CD63 and CCR3 in CD63 and CCR3 double positive cells when incubating with passively sensitized basophils (by sera from American CR allergy). Both iPer a 5 and bPer a 5 had no effect on the basophils sensitized by the sera from HC (Figure 7).

\section{Discussion}

Aerosolized proteins derived from saliva, fecal material, secretions, cast skins, debris, and dead bodies of cockroaches induce IgE-mediated hypersensitivity [24]. To better understand the Per a 5 mediated CR allergies and promise to improve diagnosis and treatment of CR allergies, we prepared biologically active and highly pure American CR allergen Per a 5 in relatively large amount in the present study. We have identified Per a 5 as a novel American CR allergen, which is recognized by $25 \%$ of the subjects with American CR allergy.

Several allergens from German and American CR have been identified and their IgE cross-reactivity has been described. These include Bla g 1 and Per a 1 (food intake and digestion); Bla g 2 and Per a 2 (inactive aspartic protease);
Bla g 3 and Per a 3 (arylphorin-like storage protein); Bla g 4 and Per a 4 (male pheromone transport lipocalin); and Bla g 7 and Per a 7 (tropomyosin) [25]. Among CR allergens, 3 GSTs were identified from male adults of German CR by glutathione-agarose affinity chromatography including one in which $\mathrm{N}$-terminal amino acid sequence is identical to that of Bla g 5 [26]. Two IgE-reactive GSTs were detected from 25 IgE-reactive spots by proteomic analysis, and one of them was found to be Bla g 5 [27]. Recently, a Delta class GST (BgGSTD1), which has 15\% amino acid sequence identity with Bla g 5, was purified from the German CR [28]. However, the allergenicity and IgE cross-reactivity of the GST homologues in American CR have not been reported. As a member of the Delta class GSTs, Per a 5 shows $81 \%$ sequence similarity to BGGSTD1, but only $13 \%$ similarity to Bla g 5 , a Sigma class GST identified from German CR. In the present study, only $25 \% \operatorname{IgE}$ reactivity to rPer a 5 was observed when sera from cockroach-sensitive patients was incubated with rPer a 5, suggesting that Per a 5 is not likely a major allergen in American CR. The IgE reactivity rate of Per a 5 seems lower than that of Bla g 5 (30 to 71\%) [12-14], but higher than that of BGGSTD1 (17.9\%) [28]. It has previously been reported that American CR extracts could not inhibit IgE antibody binding to Bla g 5 using an RIA assay, suggesting that there was no significant IgE cross-reactivity between GSTs from these two species [12]. However, Huang et al. found that GST(s) in American CR is(are) allergenic and is(are) pan-allergens in 


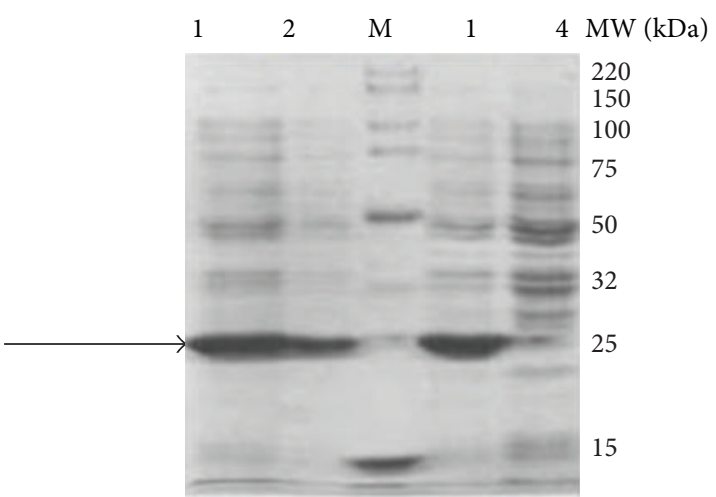

(a)

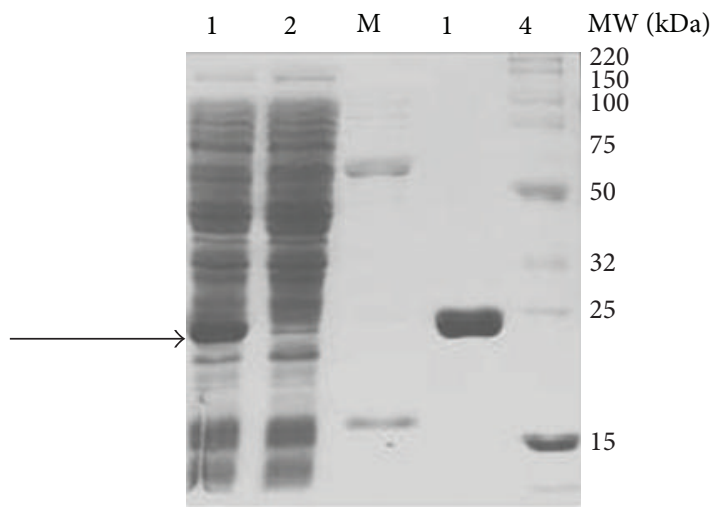

(b)

FIgURE 3: Expression and purification of Per a 5 in E. coli. (a) Per a 5 was induced by $0.1,0.5$, and $1 \mathrm{mM}$ IPTG, respectively, and was analyzed by SDS-PAGE. Lane M: Smart Broad-Range Protein Standard (Genscript, Nanjing, China); lanes 1, 2, 3, and 4 represent lysates of cells induced with $0.5,0.1,1.0$, or 0 mM IPTG, respectively. The arrow indicates Per a 5 protein. (b) SDS-PAGE analysis of purified Per a 5 expressed in E. coli. Lane M: protein standard; lane 1: the supernatant of cells lysate after centrifugation; lane 2: run through; lane 3: wash with $50 \mathrm{mM}$ imidazole; lane 4: wash with $250 \mathrm{mM}$ imidazole. The arrow indicates recombinant Per a 5 protein.

CR and mites [29]. The finding that Per a 5 reacts with specific $\mathrm{IgE}$ of American CR allergy in the present study suggests that GST in American CR is allergenic.

We prepared rPer a 5 by using two different expression systems in the present study and found that as little as $2 \mathrm{~L}$ of $E$. coli and Sf- 9 cell culture medium was able to produce 8 and $16 \mathrm{mg}$ of highly pure rPer a 5 , respectively, which is enough for functional study of Per a 5 . The E. coli system is a well-established system offering many advantages: easy handling of the bacteria cells and selection of a large variety of vectors using different promoters. Among the disadvantages, overexpressed proteins can be incorrectly folded and may require chemical refolding procedures to obtain the protein in a native, fully active, biological form. In the present study, we chose pCold II vector and E. coli Origami host strain to produce bPer a 5 in a soluble form without any reconstitution process. The eukaryotic baculovirus expression system is characterized by an extensive array of posttranslational

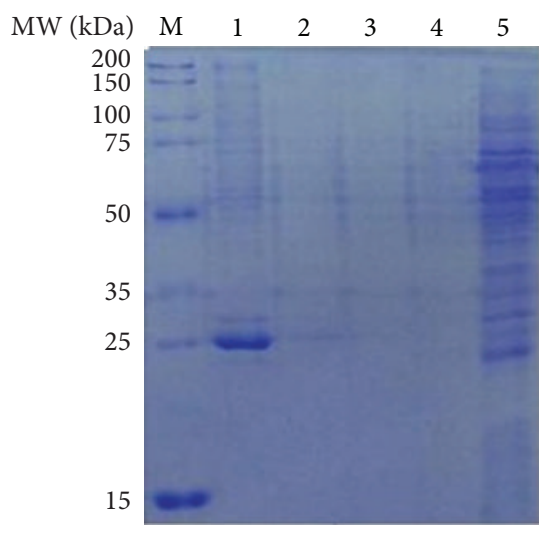

FIGURE 4: SDS-PAGE analysis of purification of Per a 5 in baculovirus-infected insect cells through Ni column. Lane M: protein standard; lane 1: elute with the elution buffer containing $250 \mathrm{mM}$ imidazole; lane 2: elute with the elution buffer containing $20 \mathrm{mM}$ imidazole; lane 3: elute with the elution buffer containing $50 \mathrm{mM}$ imidazole; lane 4: run through; lane 5: the cytosol.

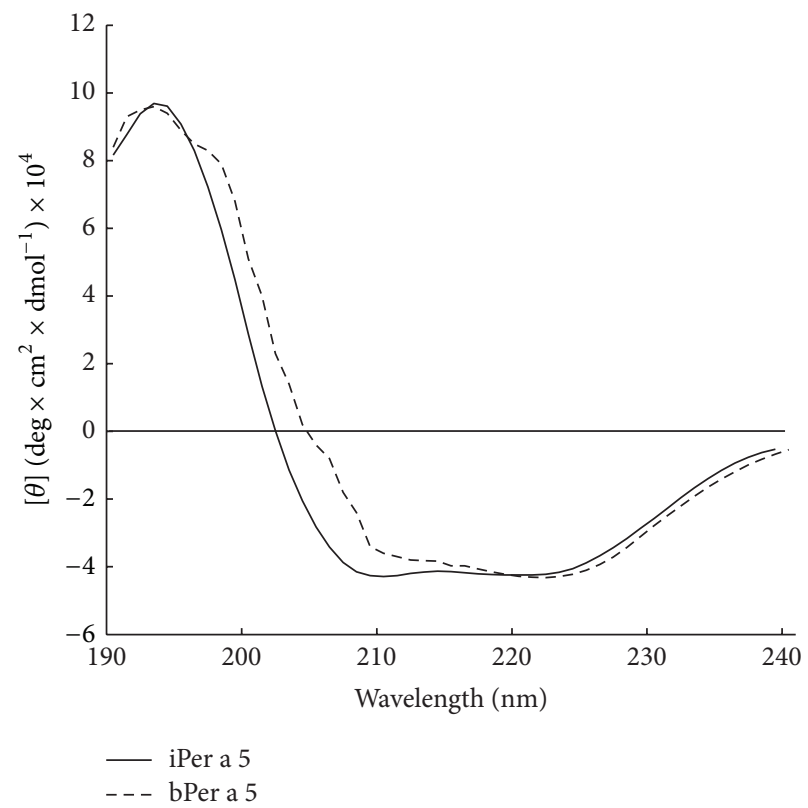

FIgure 5: Circular dichroism analysis of recombinant Per a 5 generated from baculovirus-infected insect cells (iPer a 5) and $E$. coli (bPer a 5). The molecular ellipticities $(y)$ ( $y$-axes) at different wavelengths (190-240 nm, $x$-axis) are displayed for iPer a 5 and bPer a 5 .

processing, typical for higher eukaryotic cells. The production of recombinant proteins in this system offers the advantage that secreted proteins are often glycosylated and disulphide-bonded correctly leading to a biologically active conformation. Because of the advantage of baculovirusinfected insect cell expression system, we employed it to express iPer a 5 and managed to obtain substantial quantity of iPer a 5 in the present study. Numerous insect allergens such as Api m 1 [30], Api m 2 [31] from honeybee venom, Sol i 3 [32] from ant venom, Dol m 5 [33] from baldfaced hornet (Dolichovespula maculate), Cul s 1 [34] from 

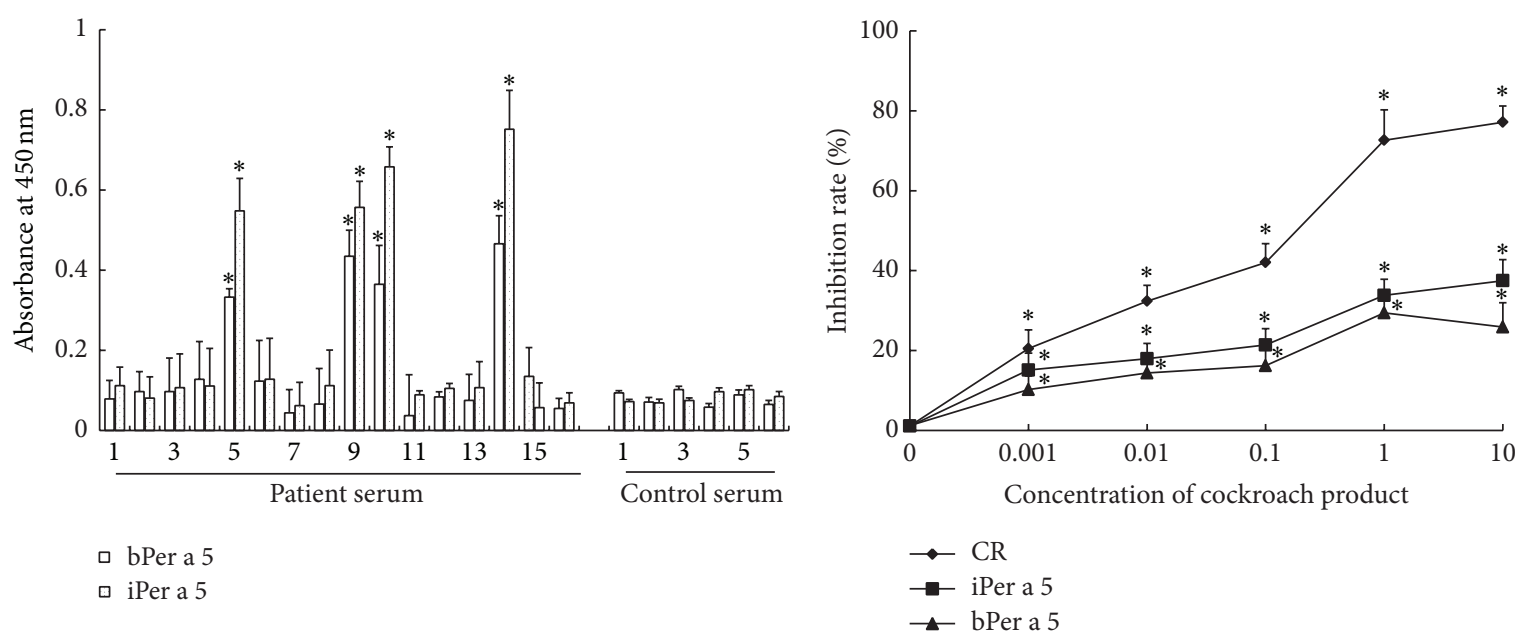

(a)

(b)

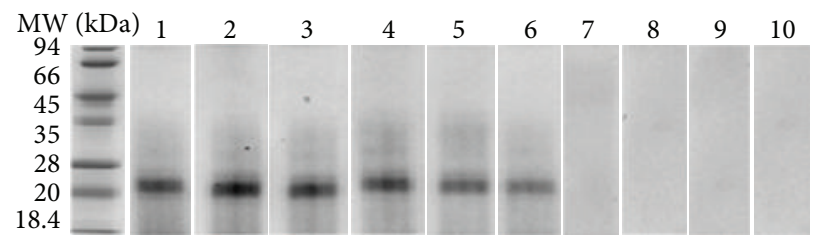

(c)

Figure 6: (a) Analysis of specific IgE reactivity of recombinant bPer a 5 and iPer a 5 by direct ELISA. The sera were collected from the patients with American CR allergy and healthy control subjects. The values shown are mean \pm SEM for the triplicate experiments. ${ }^{*} P<0.05$ in comparison with those of healthy control subjects. (b) Inhibition of the IgE reactivity to the cockroach extract by bPer a 5 and iPer a 5 . CR $=$ American cockroach extract. The values shown are mean \pm SEM for the triplicate experiments. ${ }^{*} P<0.05$ in comparison with the uninhibited control. (c) Western blot analysis of IgE reactivity to bPer a 5 and iPer a 5 in the sera from the patients with American CR allergy. Lanes 1-3: iPer a 5 reacted with the serum from patients 5, 9, and 10. Lanes 4-6: bPer a 5 reacted with the serum from patients 5, 9, and 10. Lanes 7-8: iPer a 5 reacted with the serum from controls 1 and 2. Lanes 9-10: bPer a 5 reacted with the serum from controls 1 and 2.

the North American midge (Culicoides sonorensis), Der f 1 [35] from house dust mite, Blo $\mathrm{t} 11$ [36] from dust mite (Blomia tropicalis), Lep d 2 [15] from dust mite (Lepidoglyphus destructor), and Aed a 1 and Aed a 2 [37] from mosquito (Aedes aegypti) have been successfully expressed in insect cells using a baculovirus expression system. They are reported to possess similar structural and biological activities to their natural forms [38].

Regarding the immunoreactivity, rPer a 5 produced in the two systems showed IgE-binding activities towards the sera from the American CR allergic patients. iPer a 5 seems to have better binding activity than bPer a 5, implicating that the IgE-binding activity of rPer a 5 is likely to associate with its glycosylation sites and tertiary conformation. One motif (198NHSG201) was predicted to be the glycosylation motif in Per a 5, but we are not sure of the numbers of $\alpha$ Gals in it, as most of the carbohydrate epitopes are known to lead false-positive reactions except $\alpha$-Gal [39]. The far UV CD spectra of recombinant iPer a 5 and bPer a 5 showed similar secondary structures, which consist of predominantly $\alpha$-helical structure. But iPer a 5 appears to have more $\alpha$-helical structures than bPer a 5 . IgE-binding epitopes recognized by IgE antibodies are either linear or conformational and are located on the surface of the molecule accessible to antibodies [40]. Conformational epitope comprises amino acids that are close in space in the folded molecule, despite being noncontiguous in the amino acid sequence. They are dependent on the 3-dimensional structure of the protein. For globular inhaled allergens, conformational epitopes play a very important role in allergenicity [41, 42]. Therefore, it seems likely that extra conformational IgE-binding epitopes may exist in iPer a 5, as a result of more $\alpha$-helical structures in its molecular structure.

The basophil activation test we employed herein is a more advanced technique for determination of allergenicity of a given compound. We confirm that Per a 5 is an active allergen of CR as it is able to activate CR-sensitized basophils. Similar to IgE-binding activity, iPer a 5 seems more potent in activation of basophils compared with bPer a 5, suggesting that the basophil-activating activity of rPer a 5 is likely to associate with its glycosylation sites and tertiary conformation. Obviously, further work is required to confirm our anticipation.

The availability of recombinant allergens has increased our understanding of IgE-mediated allergies and promises to improve diagnosis and treatment of these diseases [43]. In our case, rPer a 5 should be a useful tool for functional and clinical study. These observations also confirm that 

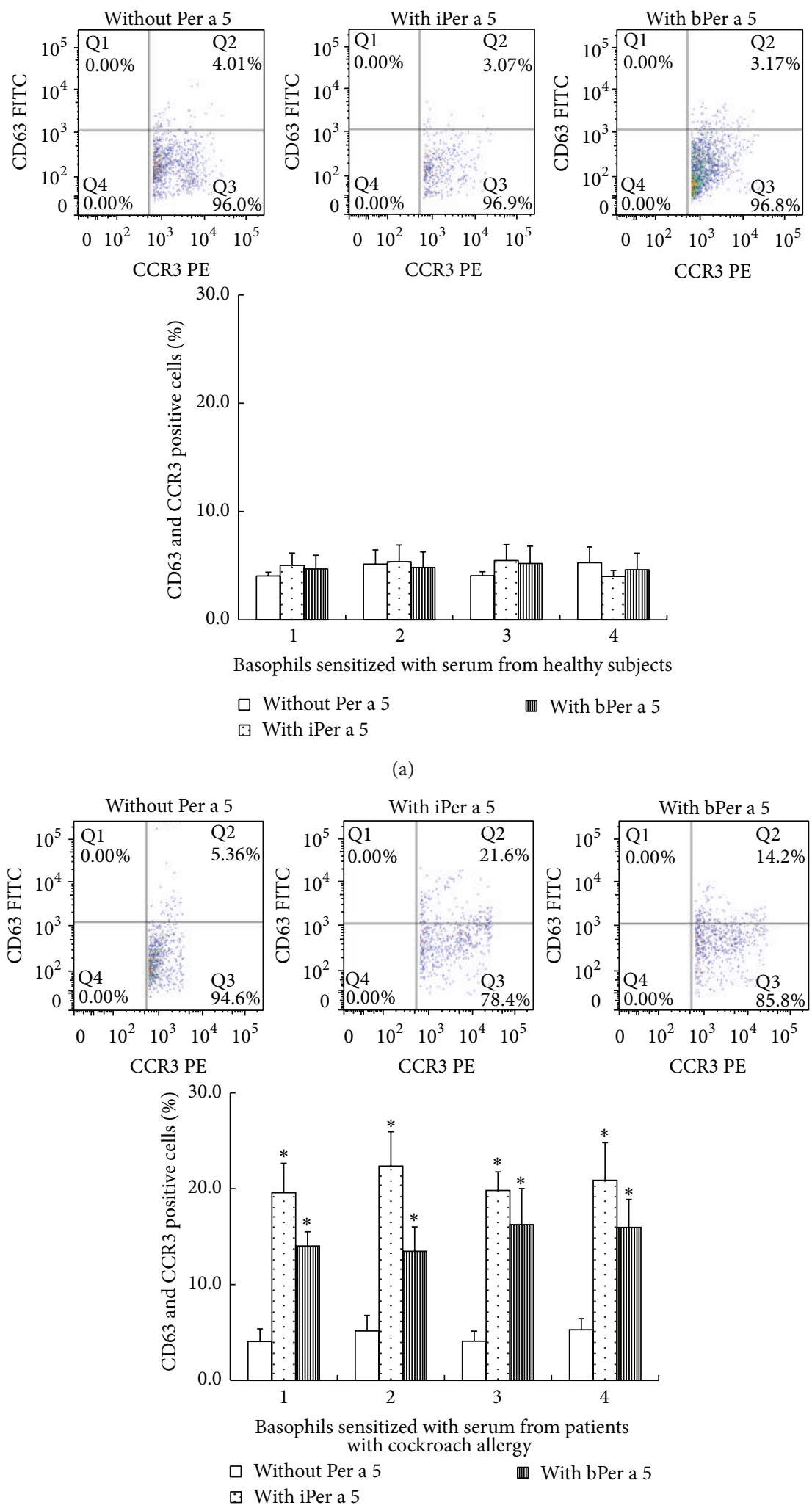

(b)

Figure 7: Induction of basophil activation by iPer a 5 and bPer a 5. After nonspecific IgE on basophils being stripped off, cells from each donor were passively sensitized with sera from 4 different healthy subjects (a) or from 4 different patients with American CR allergy (b) and were then challenged with iPer a 5 and bPer a 5 at $1.0 \mu \mathrm{g} / \mathrm{mL}$. The values shown are mean \pm SEM for the sera from 4 different subjects. ${ }^{*} P<0.05$ in comparison with the corresponding carrier alone control. 
baculovirus-infected insect cell expression system is more suitable for the production of more active Per a 5 allergen than E. coli expression system. In conclusion, we have cloned and prepared two rPer a 5 allergens by using a eukaryotic and a prokaryotic expression system. We confirm that there is GST allergen (Per a 5) in American CR, though it is not a major allergen. rPer a 5 should be a useful tool for studying and understanding the role of Per a 5 in CR allergy.

\section{Conflict of Interests}

The authors declare that there is no conflict of interests regarding the publication of this paper.

\section{Authors' Contribution}

Ji-Fu Wei and Haiwei Yang contributed to this paper equally as the first author.

\section{Acknowledgments}

This project was sponsored by the Grants from the Major State Basic Research Program of China (973 Program) (no. 2013CB530501), the National Natural Science Foundation of China (31340073, 81273274, 81172836, 30972822, 81001329, and 81030054), Jiangsu Province's Key Provincial Talents Program (RC201170), The Priority Academic Program Development of Jiangsu Higher Education Institutions (PAPD), National “Twelfth Five-Year" Plan for Science \& Technology Support Project (2014BAI07B02), the Innovation team project of Education Department of Liaoning Province (LT2013017), the higher Education Climb Scholars Program of Liaoning Province, China (no. LJ2013222), and the Liaoning Province Translational Medicine Research Center for Allergy (no. LK2013041).

\section{References}

[1] H. S. Bernton and H. Brown, "Insect allergy-preliminary studies of the cockroach," Journal of Allergy, vol. 35, no. 6, pp. 506-513, 1964.

[2] L. K. Arruda, L. D. Vailes, V. P. L. Ferriani, A. B. R. Santos, A. Pomés, and M. D. Chapman, "Cockroach allergens and asthma," Journal of Allergy and Clinical Immunology, vol. 107, no. 3, pp. 419-428, 2001.

[3] B.-Q. Sun, X.-X. Lai, B. Gjesing, M. D. Spangfort, and N.-S. Zhong, "Prevalence of sensitivity to cockroach allergens and IgE cross-reactivity between cockroach and house dust mite allergens in Chinese patients with allergic rhinitis and asthma," Chinese Medical Journal, vol. 123, no. 24, pp. 3540-3544, 2010.

[4] V. T. Sudha, N. Arora, S. Sridhara, S. N. Gaur, and B. P. Singh, "Biopotency and identification of allergenic proteins in Periplaneta americana extract for clinical applications," Biologicals, vol. 35, no. 2, pp. 131-137, 2007.

[5] S. He, Z. Zhang, H. Zhang et al., "Analysis of properties and proinflammatory functions of cockroach allergens per a 1.01s," Scandinavian Journal of Immunology, vol. 74, no. 3, pp. 288-295, 2011.
[6] Q. Pan, S. Wang, H. Shang, and F. Chew, "Identification and characterization of Per a 2, the Bla g 2 allergen homologue from American cockroach (Periplaneta americana)," Journal of Allergy and Clinical Immunology, vol. 117, no. 2, p. S115, 2006.

[7] B. Mindykowski, E. Jaenicke, S. Tenzer et al., "Cockroach allergens Per a 3 are oligomers," Developmental and Comparative Immunology, vol. 34, no. 7, pp. 722-733, 2010.

[8] Y. W. Tan, S. L. Chan, T. C. Ong et al., "Structures of two major allergens, Bla g 4 and Per a 4, from cockroaches and their IgE binding epitopes," Journal of Biological Chemistry, vol. 284, no. 5, pp. 3148-3157, 2009.

[9] J. A. Asturias, N. Gómez-Bayón, M. C. Arilla et al., "Molecular characterization of American cockroach tropomyosin (Periplaneta americana allergen 7), a cross-reactive allergen," Journal of Immunology, vol. 162, no. 7, pp. 4342-4348, 1999.

[10] N. Sookrung, W. Chaicumpa, A. Tungtrongchitr et al., "Periplaneta americana arginine kinase as a major cockroach allergen among the patients with major cockroach allergies," Environmental Health Perspectives, vol. 114, no. 6, pp. 875-880, 2006.

[11] V. T. Sudha, N. Arora, S. N. Gaur, S. Pasha, and B. P. Singh, "Identification of a serine protease as a major allergen (Per a 10) of Periplaneta americana," Allergy, vol. 63, no. 6, pp. 768-776, 2008.

[12] L. K. Arruda, L. D. Vailes, T. A. E. Platts-Mills, M. L. Hayden, and M. D. Chapman, "Induction of IgE antibody responses by glutathione S-Transferase from the German cockroach (Blattella germanica)," Journal of Biological Chemistry, vol. 272, no. 33, pp. 20907-20912, 1997.

[13] K. Y. Jeong, K.-J. Jeong, M.-H. Yi, H. Lee, C.-S. Hong, and T.S. Yong, "Allergenicity of sigma and delta class glutathione stransferases from the german cockroach," International Archives of Allergy and Immunology, vol. 148, no. 1, pp. 59-64, 2008.

[14] S. M. Satinover, A. J. Reefer, A. Pomes, M. D. Chapman, T. A. E. Platts-Mills, and J. A. Woodfolk, "Specific IgE and IgG antibody-binding patterns to recombinant cockroach allergens," Journal of Allergy and Clinical Immunology, vol. 115, no. 4, pp. 803-809, 2005.

[15] S. Olsson, M. Van Hage-Hamsten, P. Whitley et al., "Expression of two isoforms of Lep d 2, the major allergen of Lepidoglyphus destructor, in both prokaryotic and eukaryotic systems," Clinical and Experimental Allergy, vol. 28, no. 8, pp. 984-991, 1998.

[16] D. R. O’Reilly, L. K. Miller, and V. A. Luckow, Baculovirus Expression Vectors: A Laboratory Manual, Oxford University Press, New York, NY, USA, 1992.

[17] C. Perez-Iratxeta and M. A. Andrade-Navarro, "K2D2: estimation of protein secondary structure from circular dichroism spectra," BMC Structural Biology, vol. 8, article 25, 2008.

[18] K. Y. Jeong, H. Hwang, J. Lee et al., "Allergenic characterization of tropomyosin from the dusky brown cockroach, Periplaneta fuliginosa," Clinical and Diagnostical Laboratory Immunology, vol. 11, no. 4, pp. 680-685, 2004.

[19] S. An, L. Chen, J.-F. Wei et al., "Purification and characterization of two new allergens from the venom of Vespa magnifica," PLoS One, vol. 7, no. 2, Article ID e31920, 2012.

[20] S. An, D. Ma, J. F. Wei et al., "A novel allergen Tab y 1 with inhibitory activity of platelet aggregation from salivary glands of horseflies," Allergy, vol. 66, no. 11, pp. 1420-1427, 2011.

[21] M. L. Sanz, P. M. Gamboa, I. Antépara et al., "Flow cytometric basophil activation test by detection of CD63 expression in 
patients with immediate-type reactions to betalactam antibiotics," Clinical and Experimental Allergy, vol. 32, no. 2, pp. 277286, 2002.

[22] J. Sainte-Laudy, C. Vallon, and J.-C. Guerin, "Analysis of the CD63 membrane expression by human basophil activation. Applications to allergologic diagnosis," Allergie et Immunologie, vol. 26, no. 6, pp. 211-214, 1994.

[23] J. J. Pruzansky, L. C. Grammer, R. Patterson, and M. Roberts, "Dissociation of IgE from receptors on human basophils. I. Enhanced passive sensitization for histamine release," The Journal of Immunology, vol. 131, no. 4, pp. 1949-1953, 1983.

[24] P. A. Eggleston, "Cockroach allergen abatement: the good, the bad, and the ugly," Journal of Allergy and Clinical Immunology, vol. 112, no. 2, pp. 265-267, 2003.

[25] J. C. Gore and C. Schal, "Cockroach allergen biology and mitigation in the indoor environment," Annual Review of Entomology, vol. 52, pp. 439-463, 2007.

[26] S. J. Yu and S. W. Huang, "Purification and characterization of glutathione S-transferases from the German cockroach, Blattella germanica (L.)," Pesticide Biochemistry and Physiology, vol. 67, no. 1, pp. 36-45, 2000.

[27] P. T. Duong and F. N. Chang, "A simple method for assigning multiple immunogens to their protein on a two-dimensional blot and its application to asthma-causing allergens," Electrophoresis, vol. 22, no. 10, pp. 2098-2102, 2001.

[28] B. Ma and F. N. Chang, "Purification and cloning of a delta class glutathione S-transferase displaying high peroxidase activity isolated from the German cockroach Blattella germanica," The FEBS Journal, vol. 274, no. 7, pp. 1793-1803, 2007.

[29] C. H. Huang, L. M. Liew, K. W. Mah, I. C. Kuo, B. W. Lee, and K. Y. Chua, "Characterization of glutathione S-transferase from dust mite, Der p 8 and its immunoglobulin E crossreactivity with cockroach glutathione S-transferase," Clinical and Experimental Allergy, vol. 36, no. 3, pp. 369-376, 2006.

[30] S. Blank, Y. Michel, H. Seismann et al., "Evaluation of different glycoforms of honeybee venom major allergen phospholipase A2 (Api m 1) produced in insect cells," Protein \& Peptide Letters, vol. 18, no. 4, pp. 415-422, 2011.

[31] L. N. Soldatova, C. Tsai, E. Dobrovolskaia, Z. MarkovićHousley, and J. E. Slater, "Characterization of the N-glycans of recombinant bee venom hyaluronidase (Api $\mathrm{m}$ 2) expressed in insect cells," Allergy and Asthma Proceedings, vol. 28, no. 2, pp. 210-215, 2007.

[32] A. S. Borer, P. Wassmann, M. Schmidt et al., "Crystal structure of Sol i 2: a major allergen from fire ant venom," Journal of Molecular Biology, vol. 415, no. 4, pp. 635-648, 2012.

[33] M. D. Tomalski, T. P. King, and L. K. Miller, "Expression of hornet genes encoding venom allergen antigen 5 in insects," Archives of insect biochemistry and physiology, vol. 22, no. 3-4, pp. 303-313, 1993.

[34] K. F. A. Langner, D. L. Jarvis, M. Nimtz et al., "Identification, expression and characterisation of a major salivary allergen $(\mathrm{Cul}$ s 1) of the biting midge Culicoides sonorensis relevant for summer eczema in horses," International Journal for Parasitology, vol. 39, no. 2, pp. 243-250, 2009.

[35] H. Shoji, M. Hanawa, I. Shibuya et al., "Production of recombinant mite allergen der fI in insect cells and characterization of products: removal of pro-sequence is essential to IgE-binding activity," Bioscience, Biotechnology and Biochemistry, vol. 60, no. 4, pp. 621-625, 1996.

[36] A. S. M. Teo, J. D. A. Ramos, B. W. Lee, N. Cheong, and K. Y. Chua, "Expression of the Blomia tropicalis paramyosin Blo $t 11$ and its immunodominant peptide in insect cells," Biotechnology and Applied Biochemistry, vol. 45, no. 1, pp. 17-24, 2006.

[37] Z. Peng, W. Xu, H. Lam, L. Cheng, A. A. James, and F. E. R. Simons, "A new recombinant mosquito salivary allergen, rAed a 2: allergenicity, clinical relevance, and cross-reactivity," Allergy, vol. 61, no. 4, pp. 485-490, 2006.

[38] M. D. Chapman, A. M. Smith, L. D. Vailes, L. K. Arruda, V. Dhanaraj, and A. Pomés, "Recombinant allergens for diagnosis and therapy of allergic disease," Journal of Allergy and Clinical Immunology, vol. 106, no. 3, pp. 409-418, 2000.

[39] H. Malandain, "IgE-reactive carbohydrate epitopes-classification, cross-reactivity, and clinical impact," European Annals of Allergy and Clinical Immunology, vol. 37, no. 4, pp. 122-128, 2005.

[40] A. Pomés, "Relevant B cell epitopes in allergic disease," International Archives of Allergy and Immunology, vol. 152, no. 1, pp. $1-11,2010$.

[41] T. Midoro-Horiuti, V. Mathura, C. H. Schein et al., "Major linear IgE epitopes of mountain cedar pollen allergen Jun a 1 map to the pectate lyase catalytic site," Molecular Immunology, vol. 40, no. 8, pp. 555-562, 2003.

[42] S. J. Maleki, R. A. Kopper, D. S. Shin et al., "Structure of the major peanut allergen Ara h 1 may protect IgE-binding epitopes from degradation," Journal of Immunology, vol. 164, no. 11, pp. 5844-5849, 2000.

[43] M. Schmidt and D. R. Hoffman, "Expression systems for production of recombinant allergens," International Archives of Allergy and Immunology, vol. 128, no. 4, pp. 264-270, 2002. 


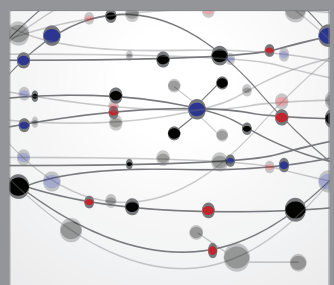

The Scientific World Journal
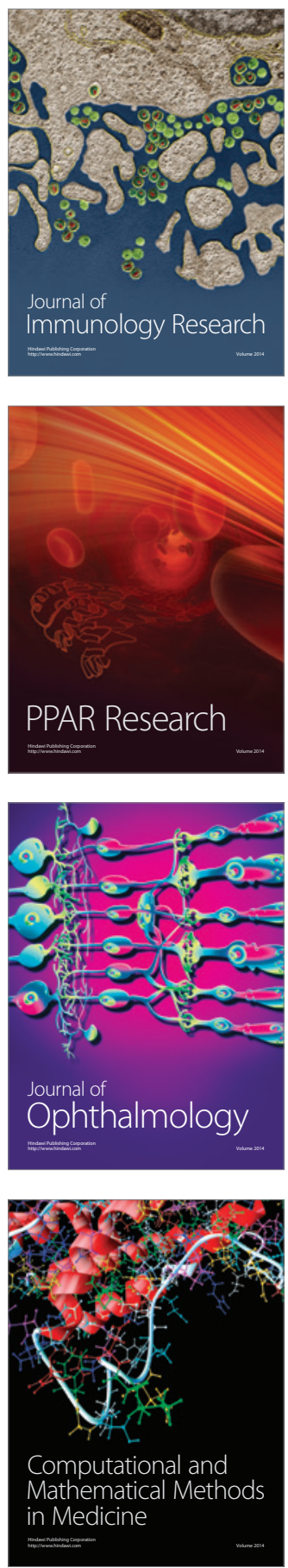

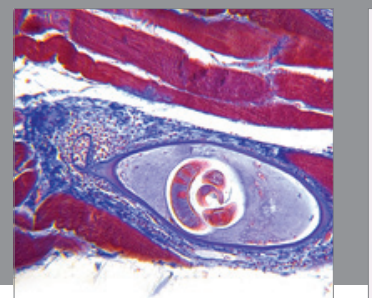

Gastroenterology

Research and Practice
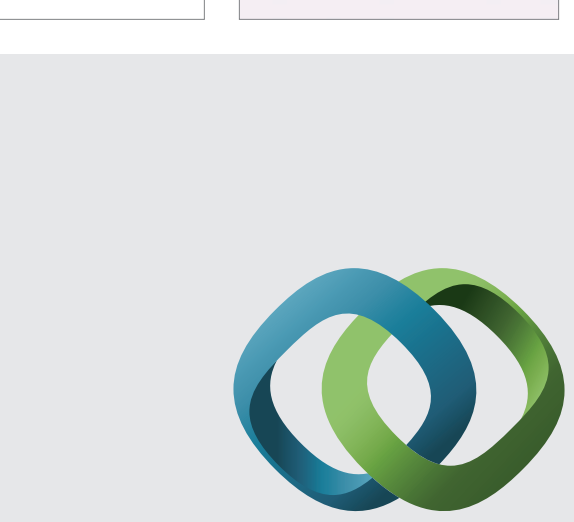

\section{Hindawi}

Submit your manuscripts at

http://www.hindawi.com
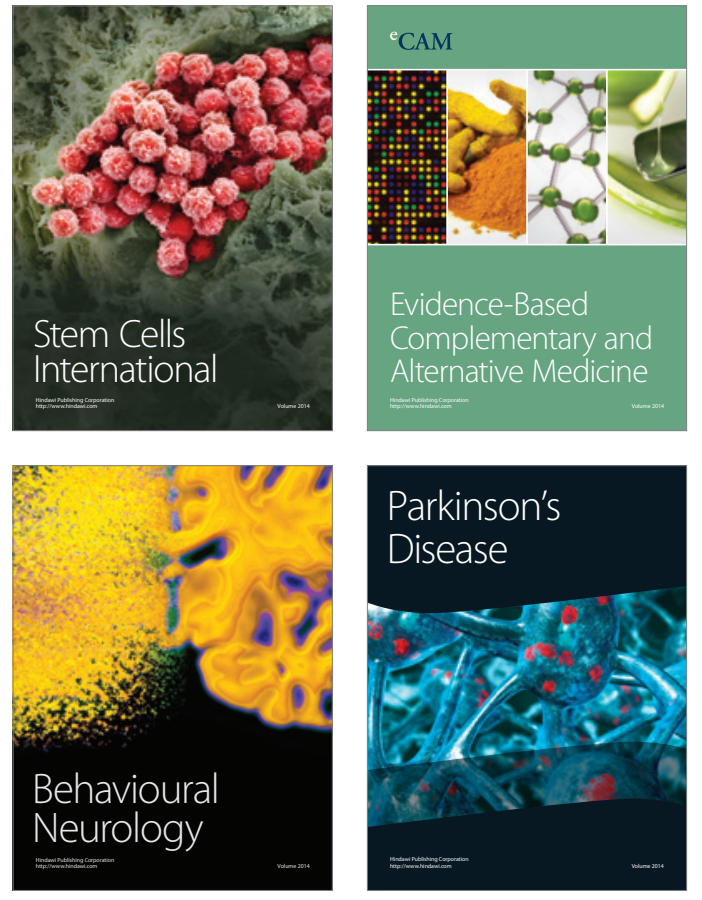
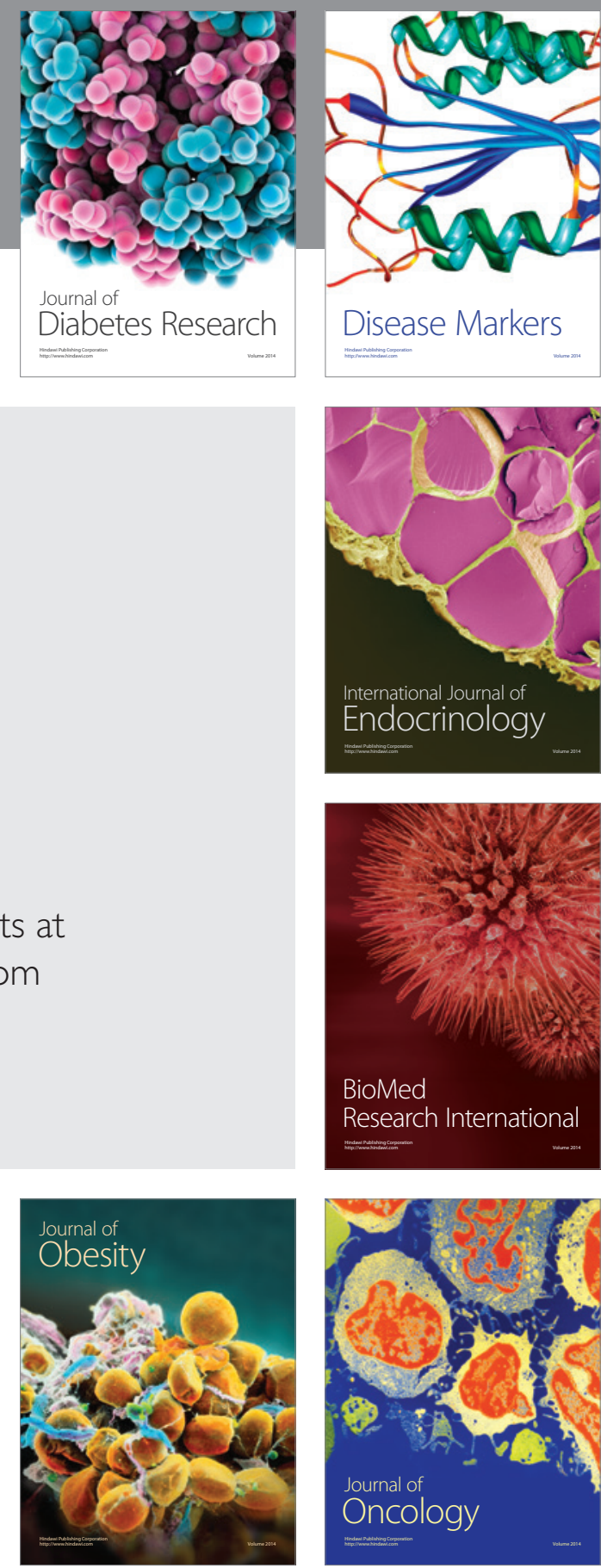

Disease Markers
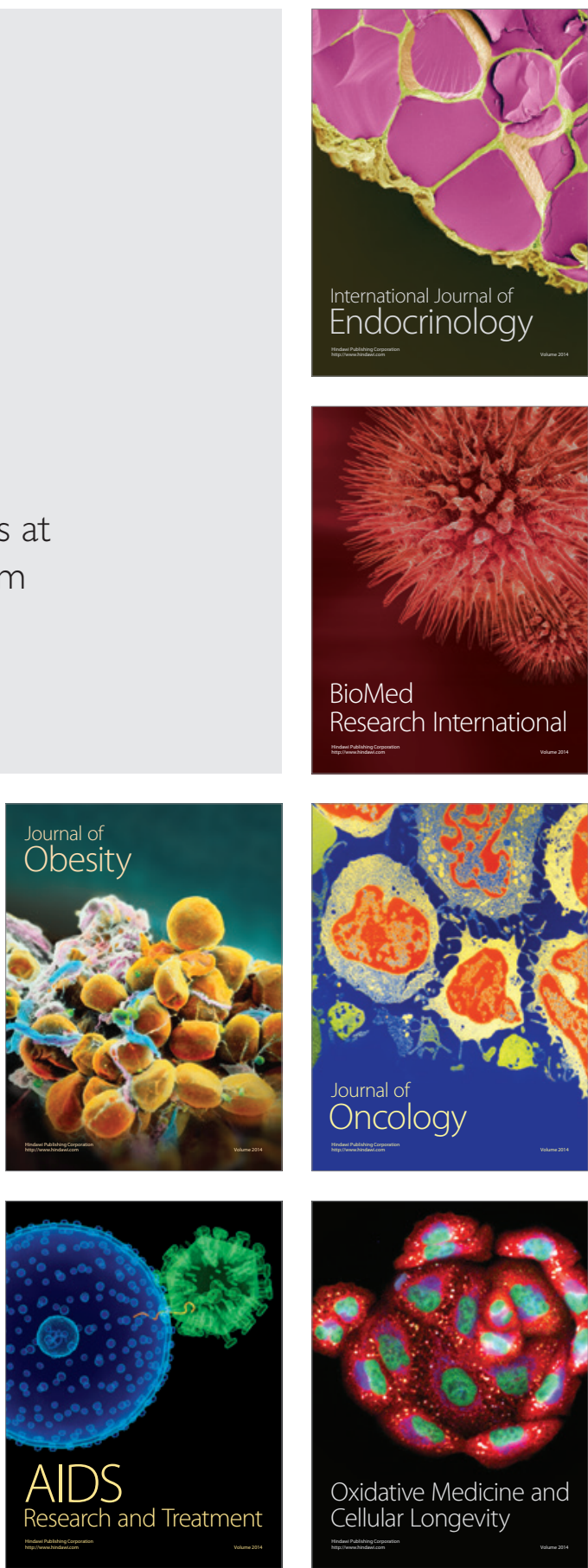